\title{
Article
}

\section{Developing clinical academic researchers: insights from practitioners and managers in nursing, midwifery and allied health}

Roddam, Hazel, Cross, Lucy, Georgiou, Rachel, Jones, Stephanie, Olive, Philippa, Smith, Grete and Thomas, Lois Helene

Available at http://clok.uclan.ac.uk/29409/

Roddam, Hazel ORCID: 0000-0002-0637-1801, Cross, Lucy ORCID: 0000-00027272-9445, Georgiou, Rachel, Jones, Stephanie ORCID: 0000-0001-91498606, Olive, Philippa ORCID: 0000-0002-9175-1285, Smith, Grete and Thomas, Lois Helene ORCID: 0000-0001-5218-6546 (2019) Developing clinical academic researchers: insights from practitioners and managers in nursing, midwifery and allied health. British Journal of Healthcare Management, 25 (9). pp. 282-292. ISSN 1358-0574

It is advisable to refer to the publisher's version if you intend to cite from the work. 10.12968/bjhc. 2019.0020

For more information about UCLan's research in this area go to http://www.uclan.ac.uk/researchgroups/ and search for <name of research Group>.

For information about Research generally at UCLan please go to http://www.uclan.ac.uk/research/

All outputs in CLoK are protected by Intellectual Property Rights law, including Copyright law. Copyright, IPR and Moral Rights for the works on this site are retained by the individual authors and/or other copyright owners. Terms and conditions for use of this material are defined in the policies page. 
Developing clinical academic researchers in practice: insights from practitioners and managers in nursing, midwifery and allied health.

Hazel Roddam, Lucy Cross, Rachel Georgiou, Josephine Gibson, Stephanie Jones, Philippa Olive, Grete Smith, Lois Thomas

University of Central Lancashire (UCLan)

Corresponding author: Dr Hazel Roddam School of Health Sciences, University of Central Lancashire HRoddam@uclan.ac.uk

\section{Abstract}

Background: Developing a clinical academic role in Nursing, Midwifery and the Allied Health Professions (NMAHP) is challenging due to lack of a national career pathway, recognition and understanding of the role.

Aims: This evaluation explored perspectives of aspiring or active clinical academics and health care managers in NMAHP about the benefits, barriers and enablers of engagement in these career pathways.

Methods: Eight workshops were facilitated across England (four each for managers and prospective clinical academics); 162 participants shared their experiences and perceptions of clinical academic research activities.

Findings: Three major themes were identified related to the perceived benefits, barriers and enablers of engagement in these career pathways: Building health research capacity; Building individuals' health research capability; and Improving patient care. 
Conclusion: This report demonstrates factors that are valued and perceived to be working well by practitioners and their clinical service managers, and highlights key priorities for further strategic support.

\section{Acknowledgement}

The authors wish to thank the following organisations for their support and input to these workshops: Health Education England (HEE), NIHR Trainees, Research Design Service (RDS), Clinical Research Network (CRN), Council for Allied Health Professions Research (CAHPR).

We also thank all the workshop participants who generously shared their experiences, insights and comments for this evaluation.

\section{Conflict of interest statement}

The authors declare that they have no conflict of interest in this work.

Funding for the workshops was provided by Health Education England to promote the uptake of the NIHR Integrated Clinical Academic pathway. JG is partly funded by the National Institute for Health Research Collaboration for Leadership in Applied Health Research and Care North West Coast. The views expressed are those of the authors and not necessarily those of the NIHR, NHS, Department of Health and Social Care, or Health Education England. 
Clinical academic research in NMAHP

Developing clinical academic researchers in practice: insights from practitioners and managers in nursing, midwifery and allied health.

\section{Introduction}

Health care organisations with research-active staff have improved processes of care, better patient-reported experiences of care, and may also have improved patient outcomes (Boaz et al 2015; Ozdemir et al., 2015). Organisational engagement in research is strongly associated with innovation and quality improvement (Jabbal, 2017) and engagement in research is also supported by the NHS Constitution for England (2015 p3). Supporting the growth of clinical academic research careers in nursing, midwifery and the allied health professions (NMAHPs) is therefore key to the delivery innovative, world-class health care.

An NMAHP Clinical Academic has been defined as a nurse, midwife or allied health professional who engages concurrently in clinical practice and research:

"a successful clinical academic will be able to demonstrate not only that they are an excellent researcher but also that they can lead and inspire others in the clinical field" (AUKUH 2016 p9).

These roles enable research that is directly informed by and relevant to clinical practice and allow practitioners to follow a research career whilst optimising their clinical expertise (AUKUH, 2016; CAHPR 2018). 
NMAHP researchers have a long history of contributing to research and translation of highquality evidence into clinical practice (DH 2012). However, developing a clinical academic role in NMAHPs has been challenging due to the lack of a national career pathway, recognition and understanding of the role (Pickstone et al 2008). In contrast to the wellestablished clinical academic career route in medicine comprising nearly $5 \%$ of consultants, only $0.1 \%$ of NMAHPS in the NHS have formal clinical academic posts (Baltruks and Callaghan, 2018).

To address this shortfall, a national clinical academic training pathway was established in 2008 (NIHR 2018) ${ }^{1}$, and numerous local and regional initiatives have subsequently emerged. Despite consultation exercises with senior leaders such as NMAHP directors and senior academics (AUKUH 2016), little is known about frontline practitioners' and managers' views of the barriers and enablers to these new clinical academic roles.

The aim of this evaluation was to explore the perspectives of health care managers and of aspiring or active clinical academics in nursing, midwifery and allied health professions, about the benefits, barriers and enablers of engagement in these career pathways. The findings reported in this article are based on our experiences of delivering workshops across England to promote uptake and support for clinical academic career pathways, as well as our extensive experience in teaching and supporting research engagement and leadership in

\footnotetext{
${ }^{1}$ The HEE/NIHR Integrated Clinical Academic Programme (ICA) is a partnership between Health Education England and the National Institute for Health Research. This initiative aims to provide career pathways for nonmedical health professionals in England, that comprise incremental development of academic and leadership skills in clinical practice settings. The rationale is that a diverse, multi-professional clinical academic workforce is needed to address the full range of research questions that matter to patients. https://www.nihr.ac.uk/ourresearch-community/NIHR-academy/nihr-training-programmes/nihr-hee-ica-programme/
} 
healthcare, and of being research-active practitioners ourselves in nursing (JG, PO, RG) and allied health (HR).

\section{Methods}

The evaluation drew on feedback from 162 delegates at eight full-day workshops facilitated by the authors in early 2017. In each of four regions across England we held two workshops, one for managers and one for prospective clinical academics. These free workshops had been widely publicised as being open to all registered NMAHP professionals working in any roles. Due to the tight 12 -week timeline of the project it was not possible to arrange a purposive selection of participants, but the profile of all delegates was analysed across each region, to evaluate the spread of healthcare disciplines represented.

All workshops followed a common agenda to balance information dissemination with active participation by delegates. We captured feedback from delegates before, during and after the workshops via a variety of sources (Table 1 shows the overview of all data collected). Participants' priority information and support requests were collected through an anonymous post-it note exercise $(n=500+)$. A group consensus of reported perceptions of local and regional assets and opportunities, and proposed development needs was captured in note form as a flipchart exercise. During the workshops, we also invited all delegates to record written advice that they would like to share with colleagues about clinical academic research: this "soundbite studio" activity generated over 250 anonymous 'speech bubble' written messages. 
All the data sources were reviewed separately for each workshop event, then for each region, and then combined for all events. The items generated through the consensus group exercises were used as a basis for comparison of recurrent issues, related to key opportunities and threats for sustainable clinical academic research. Four members of the project team completed an iterative thematic analysis process (based on Attride-Stirling, 2001), incorporating all the basic and organising themes into 3 over-arching global themes. These were later verified by the full team.

(Insert Table 1 here)

\section{Ethical considerations:}

All delegates were informed verbally at the beginning of the workshop that their comments and feedback would be used in anonymised form for reporting and dissemination. After the end of the project we obtained a favourable ethical opinion from the host university and explicit agreement from NIHR as the project sponsor, for publication of a fully anonymised report of the workshop findings (Reference: STEMH 728).

\section{Findings}

Three major themes were identified relating to the perceived benefits, barriers and enablers of engagement in these career pathways: 1) Building organisational health research capacity; 2) Building individuals' health research capability; and 3) Improving patient care. 
Figure 1 illustrates the significance of these distinctive and complementary themes, and Table 2 lists the specified strategies proposed within each theme.

(Insert Figure 1 here)

(Insert Table 2 here)

\section{1) Building organisational research capacity}

Participants identified improvements in infrastructure and resources needed to enable clinical academic career pathways, such as regional and national co-ordination of funding schemes and support, as well as specific support for Research and Development departments to enable them to assist practitioners applying for such schemes. Participants stressed that support should include both NIHR and other funding routes at all levels. The aim should be to increase clinical NMAHPs' capacity to lead research, rather than solely being collaborators in research led by medical colleagues or non-clinical researchers.

Alternative pathways and roles were suggested, including 'home-grown' communities of clinical academics via local or regional initiatives with protected clinical research time. Joint NHS-HEI appointments also had potential to provide sustainable career paths and to maximise the contribution of practitioners' experience and expertise without enforcing a career choice between clinical practice, teaching and research. Bridging funds for clinicians at pre-doctoral or early post-doctoral level were suggested to support the development of fellowship or other funding applications. Opportunities could be promoted and supported by local and regional Research Design Services and Clinical Research Networks. Even small grants could have significant impact, such as writing retreats and small funding awards ringfenced for clinical practitioners as novice researchers. All opportunities needed to be 
Clinical academic research in NMAHP

widely publicised, including sharing success stories and case studies within and between organisations and professional disciplines:

"Increased dissemination and visibility [of information] is key to inform those pursuing and hosting research." (Participant, Workshop 3)

Existing support networks included clinical academic schemes and research networks between universities and hospitals. Practitioners working in fields or localities with less welldeveloped research networks reported facing greater barriers to pursuing these pathways. Development of regional inter-professional collaborations was recommended for supporting smaller professional discipline groups.

Trusts could foster potential clinical academic researchers, for example by inviting expressions of interest to write fellowship and grant applications at junior and senior entry points, rather than waiting for individuals to request support. Raising awareness for practitioners and managers could "sell the benefits" of clinical academic careers to the host NHS organisations:

"Needs more promotion with middle managers in NHS so the value of research-active clinicians can be appreciated more". (Participant, Workshop 7)

Training and support for clinical managers is essential to ensure that they value and understand how they could facilitate clinical staff to engage in research:

"Don't forget the managers that have to support staff - manage their expectations, manage the vacancy and the challenges of backfill. We need help and support to support our staff." (Participant, Workshop 3) 
Part-time working raised additional challenges in balancing research and clinical roles. Managers also voiced concerns about retaining staff in clinical practice after investing time and money to support their research training, which could be achieved by development of permanent clinical academic posts. Additional funding for bank or locum staff cover for those undertaking research skills training could address some concerns about the capacity of health services to release staff to undertake research activity.

More explicit remits for clinical research in job descriptions and appraisals was needed. Managers themselves also needed time to effectively support research-active staff, to maximise the benefits of their skills:

"Work with health employers to plan for engaging in clinical careers after MSc and PhD qualification." (Participant, Workshop 1)

\section{2) Building individuals' health research capability}

There are challenges and opportunities for all individuals contemplating or engaging in clinical academic activities. Diverse examples of successful clinical-academic career journeys need to be widely shared. Whilst the ICA pathway was felt to be "relevant to all clinical staff, regardless of career stage" (Participant, Workshop 4), many participants urged others to "start the research journey as early as possible in your career" (Participant, Workshop 2), to optimize the benefits for patient care and career development. Clinical-academic pathways could be better promoted within HEls by more actively promoting awareness of the potential for clinical-academic research activities and careers, as reflected in the Nursing and Midwifery Council's draft standards (NMC 2017), which state that "... Research careers need to be embedded in pre-reg(istration) and post-reg education models". 
Respondents reported that informal networking and mentorship were also essential to sustain motivation, describing themselves as needing "resilience" to continue. More accessible ways of locating research-active contacts and potential mentors/supervisors were requested, and social media was suggested as a way to foster regional networking, although opportunities for face-to-face networking were also essential. Models such as the national "Hub" network (Council for Allied Health Professions Research https://cahpr.csp.org.uk/) were recommended to support novice clinical academics to "learn the language" and build their understanding. Networking could also enable access to role models and mentors, to contribute to informal mentoring of individuals and teams, enable better engagement of managers, and foster clarity about the respective responsibilities of managers and academic supervisors. "Buddy" links between Trusts and HEls could formalise support routes, for example, internships and shadowing:

"Continue to support researchers beyond any formal development ... encourage mentoring/coaching of the next generation." (Participant, Workshop 3)

Novices could also be encouraged by communication with peers who were only a step ahead in their research journey: "If I can do it, you can do it too". (Participant, Workshop 8) Practitioners reported the positive influence of supportive colleagues and a research-active culture and ethos:

“... let's create a culture of research openness in practice". (Participant, Workshop 7) 
In regions where there were several University Hospitals, practitioners reported a particularly strong research ethos, but this was often focused on medically-led research and did not necessarily translate into a supportive environment for NMAHP research. Building links with existing prestigious NMAHP research groups, and 'bottom up' activities such as journal clubs and writing workshops, to develop a critical mass of multi-professional research-engaged practitioners were felt to be important to "develop power in numbers" (Participant, Workshop 3)

Participants who had undergone some clinical academic training reported that it had helped them to develop a self-driven, resourceful approach, satisfying their intellectual curiosity and passion for research, whilst still continuing to work in clinical practice. Some also reported that they had developed entrepreneurial and innovation skills, which were of added value to their teams and organisations. Training opened up diverse career pathways which combined roles in practice, management, research and teaching. These professionally challenging and satisfying career opportunities helped to retain and maximise talents within the healthcare workforce. Avoiding having to make an 'either - or' choice meant that talented staff were not lost from frontline healthcare into academia:

"Develop yourself, your career and improve patient care" (Participant, Workshop 6)

\section{Improving patient care}


This included two complementary themes: developing evidence-based practice and conducting practice-based research. Building research awareness, skills and activity in the healthcare workforce would itself improve patient care via the implementation of research findings in practice:

"building and developing a research culture within clinical practice is vital for developing best practice \& evidence-based practice" (Participant, Workshop 2)

It was felt that engagement in clinical research should be the norm rather than the exception, involving the whole department or clinical team to support development of a positive research culture. Participants also felt that clinical academics were best placed to contribute to and lead research studies which addressed questions that were grounded in genuine clinical priorities and perspectives. This would help to move research away from biomedical models and towards diverse perspectives and methodologies, in line with current trends towards multidisciplinary patient care and professional autonomy for NMAHPs. They exhorted others to:

"take on the challenge if you believe clinical and research work should truly inform one another" (Participant, Workshop 1)

Practitioners also suggested that clinical academics could enhance engagement of patient groups in research, including recruitment to applied health care studies as well as consultation to develop co-designed research plans and priorities. This would enable research to reflect the needs and priorities of patient-centred care and to create: 
"... the opportunity to develop future practice for my patients" (Participant, Workshop 6)

Clinical academics could also "act as a bridge" between non-research active practitioners and non-practising academics by facilitating greater understanding and commitment to shared goals. It was also proposed that research could potentially improve costeffectiveness as well as clinical effectiveness, but this added value needed to be evidenced, for example by case studies:

"we need to get our commissioners...on board with research as we need to work smarter with less money!" (Participant, Workshop 7)

"make sure that the outcomes it achieves are directly connected to clinical practice (and that this can be demonstrated!)" (Participant, Workshop 5)

\section{Discussion}

This study has demonstrated the perceived value of clinical academic research to practitioners and managers. Participants highlighted the potential benefit to patient care through increased awareness and ability to implement research evidence in practice. Skills highlighted as part of clinical-academic training included critical thinking, implementation of research evidence into practice, and innovation. These are perceived to benefit patients, and to provide added value to both teams and organisations. (The NIHR websites feature a 
number of case studies across NMAHP disciplines and career levels which also illustrate these benefits) ${ }^{2}$

Managers are key to 'talent spotting' and encouraging the development of clinical academic practitioners and to providing an environment in which they can thrive. To fulfil this, managers reported that they require additional relevant training and support. In particular, managers reported that even where finances are available to backfill posts to support clinical academic activities, it can be highly challenging to recruit individuals who have directly relevant expertise and who are available.

These findings support the acknowledged challenges for the integration of clinical academic activities within NMAHP service delivery. Our participant grassroots practitioners and service managers affirmed the predominant barriers identified in recent high level stakeholder consultation reports (AUKUH 2016 p18-19; Baltruks and Callaghan, 2018). However, our participants additionally emphasised that the current insecurity of these career routes poses a very significant risk to recruitment and retention of these exceptionally skilled individuals; with consequent threat to the future contribution of these roles for facilitating ongoing excellence in patient care.

\footnotetext{
${ }^{2}$ NIHR Internship examples https://healtheducationengland.sharepoint.com/Comms/MaE/Shared\%20Documents/Forms LAlltems.aspx?id=\%2FComms\%2FMaE\%2FShared\%2ODocuments\%2FProjects\%2FHEE\%20cor porate\%20website\%2FWebsite\%20content\%2FHEE\%2ONIHR\%2OIntegrated\%20Clinical\%20A cademic\%20Programme\%20case\%20studies \& $p=$ true \&cid=893c30aa-8a46-4f12-a542$\underline{8 d 5 c 87 c 810 f a}$

Video case studies across the range of NIHR Integrated Clinical Academic career levels https://www.youtube.com/playlist?list=PLrVQaAxyJE3eqWpdR2uwZwCLHJBZFqWmK
} 
The commonality of these themes across all the NMAHP groups demonstrates the originality of these findings, and the wider relevance of the implications for healthcare organisations. Other strengths of this study include the multiple data sources from the participant feedback and insights plus a relatively large sample size across all four regions. As this study was conducted in England, the findings may not necessarily be transferrable to other nations, although similar initiatives exist elsewhere (eg Scottish Government 2017).

\section{Limitations of the study}

Our cohort of participants were highly engaged with the agenda for clinical academic research, and as a self-selecting sample they may not be fully representative of perceptions of this issue across the NMAHP workforce. In addition, these findings only provide a snapshot of practitioners' and managers' current perceptions. The context is continuing to change with new pre-doctoral bridging schemes launched shortly after our workshops. These new career routes will need to be evaluated in due course (Sarre and Cooke 2009; Wenke et al 2017); although opportunities are still relatively limited in number and highly competitive.

This series of workshops was funded by Health Education England and included successful case studies from across the NMAHP disciplines and all five levels of the NIHR HEE ICAP for research development. These exemplars may have influenced some of the participants' responses; however, the workshop question prompts focused directly on delegates' 
personal experiences and local initiatives. Finally, there is the potential limitation of valuesbias in the data analysis; we aimed to mitigate this by the transparency of our processes, and the inclusion of supporting evidence from the participants' own voices.

\section{Conclusions}

These findings have direct implications and application for practice and workforce planning across all healthcare services and organisations. The findings have direct implications for practice and workforce planning across all healthcare services and organisations. Feedback from workshop participants clearly demonstrates factors that are valued and perceived to be working well by practitioners and their clinical service managers, including exemplars of where a positive research culture has been established within local services. It also highlights key priorities for further strategic support, such as more explicit promotion of the 'added value' for patient care as a direct result of clinical academic activity.

There is a need for well-designed impact evaluations of clinical academic research roles and activity, to add further robust evidence of the potential benefits, for example improved patient experience and more evidence-based processes of care. Whilst acknowledging the methodological challenges for conducting service-level evaluations and case-based studies, this could add further convincing evidence to the case for clinical academic roles

\section{KEY POINTS}


- There is high practitioner motivation to improve excellence in evidence-based patient care

- This can be best supported where there is a "critical mass"

- The key driver for NMAHP clinical academic research is to improve excellence in evidence-based patient care

- There should be a multiple opportunities and pathways for individuals (national, regional, local, uni- and multi-professional, and virtual networks) as well as maximising the potential of those in research delivery roles

Keywords clinical academic careers, health research, nursing, midwifery, allied health professions

\section{REFLECTIVE QUESTIONS}

1. How can you and your organisation 'talent spot' future NMAHP clinical academic research stars?

2. How can your organisation support NMAHP individuals at all levels of their clinical academic career pathway?

3. What are the challenges and opportunities in developing new career models to integrate clinical practice and research in NMAHPs?

\section{References}


Attride-Stirling, J. Thematic networks: an analytic tool for qualitative research (2001)

Qualitative Research, 1(3), 385-405. https://doi.org/10.1177/146879410100100307

Association of UK University Hospitals (AUKUH) Transforming healthcare through clinical academic roles in nursing, midwifery and allied health professions: A practical resource for healthcare provider organisations (2016)

https://www.england.nhs.uk/leadingchange/research-and-evidence/useful-resources/

Baltruks D. and Callaghan P. Nursing, midwifery and allied health clinical academic research careers in the UK (2018) Council of Deans of Health

Boaz A, Hanney S, Jones $T$, et al. Does the engagement of clinicians and organisations in research improve healthcare performance: a three-stage review. BMJ Open 2015;5: e009415. doi:10.1136/bmjopen-2015-009415

Council for Allied Health Professions Research (CAHPR) Top Ten Tips Enabling Research Activity 2018 https://cahpr.csp.org.uk/content/cahpr-top-ten-tips

Department of Health (2012) 'Developing the Role of the Clinical Academic Researcher in the Nursing, Midwifery and Allied Health Professions', Available 
Clinical academic research in NMAHP

at: https://www.gov.uk/government/publications/developing-the-role-of-the-clinicalacademic-researcher-in-the-nursing-midwifery-and-allied-health-professions

Jabbal, J. (2017) Embedding a culture of quality improvement. Kings Fund https://www.kingsfund.org.uk/sites/default/files/2017-11/Embedding-culture-QI-KingsFund-November-2017.pdf

National Health Service (NHS) Constitution (2015)

https://assets.publishing.service.gov.uk/government/uploads/system/uploads/attachment data/file/480482/NHS Constitution WEB.pdf

National Institute for Health Research Building a research career (2016)

https://www.nihr.ac.uk/our-faculty/documents/Building-a-research-career-handbook.pdf

National Institute for Health Research ICA HEE/NIHR Integrated Clinical Academic Programme for non-medical healthcare professions (2018)

https://www.nihr.ac.uk/funding-and-support/funding-for-training-and-careerdevelopment/training-programmes/nihr-hee-ica-programme/

Nursing and Midwifery Council (NMC) Standards of proficiency for registered nurses consultation document (2017) 
https://www.nmc.org.uk/globalassets/sitedocuments/edcons/ec7-draft-standards-ofproficiency-for-registered-nurses.pdf (accessed 4/12/17)

Ozdemir BA, Karthikesalingam A, Sinha S, et al. Research Activity and the Association with Mortality. Chalmers JD, ed. PLoS ONE. 2015;10(2):e0118253. doi:10.1371/journal.pone.0118253.

Pickstone C, Nancarrow S, Cooke J, Vernon W, Mountain G, Boyce A, Campbell J. Building research capacity in the allied health professions. Evidence and Policy. 2008 4(1) 75-91.

Sarre G, and Cooke J. Developing indicators for measuring Research Capacity Development in primary care organisations: A consensus approach using a Nominal Group Technique. Health and Social Care in the Community. 2009 17(3) 244-253

Scottish Government (2017) A clinical academic approach for nurses, midwives and allied health professionals - it's a no-brainer! http://www.nmahp-

ru.ac.uk/media/microsites/nmahp-ru/documents/Clinical-academic-approach-for-nursesmidwives--AHPs.pdf

Wenke, RJ, Ward, EC, Hickman I, Hulcombe J, Phillips R, Mickan S. (2017) Allied health research positions: a qualitative evaluation of their impact Health Research Policy and Systems 2017 15:6 
Clinical academic research in NMAHP 